\title{
British Surfers and Their Attitudes and Values Toward the Environment
}

\author{
David Holland-Smith, Aharon Love, and Ross Lorimer \\ School of Social and Health Sciences, University \\ of Abertay Dundee, UK.
}

\begin{abstract}
The purpose of this study was to examine the attitudes of British surfers toward their environment. Eight surfers were interviewed and their views analyzed using an inductive thematic analysis to explore their perceptions of the factors that have influenced their engagement in proenvironmental behaviors. The findings suggest that surfers (in this sample), while motivated to engage in proenvironmental behaviors, are also influenced by a range of factors and appear to be primarily motivated by their identification and a need to maintain their experience of that sport. This reveals an inherent dilemma. Through their sport surfers are able to observe the effects of environmental degradation on the environment (e.g., polluted water), the ecosystem, and on their personal health. However, they are also aware of the trade-offs required in pursuing their sport that may damage the environment (e.g., toxic material involved in sport kit manufacture). When questioned, surfers stated they would be unwilling to give up their sport even at the cost of the environment in which those activities are experienced. This highlights a possibly common problem among environmentally minded outdoor enthusiasts: when do personal benefits outweigh the responsibilities of environmental stewardship? The value of these findings is discussed in relation to the promotion of proenvironmental behaviors and engagement with proenvironmental groups.
\end{abstract}

URFERS ARE KNOWN to have a complex relationship with their environment, and the quality of that environment is intrinsically important to their experience of surfing (Wade, 2007). Surfing is an activity that can involve an intense embodied experience in which surfers are physically and emotionally immersed in their environment (Midol \& Broyer, 1995). Surfers spend much of their time in or near the water, and they are often among the first to develop symptoms related to water contamination. Additionally, some of the most popular surfing locations in the world are offshore at reefs that are increasingly being lost due to complex environmental factors such as increasingly warmer water temperatures and rising sea levels (Wade, 2007). It would therefore seem to be in the surfers' best interests to protect their environment. Steg and Vlek (2009) define this action as proenvironmental behavior, which is any behavior which can be seen as being beneficial to the environment.

Despite the apparent need for surfers to protect their environment, surfing activities can and do have a negative impact. Surfboards are often composed of chemicals that are considered to be extremely hazardous to the environment both during manufacturing and disposal. Wade (2007) describes them as being made of the most nonbiodegradable products on the planet with over 750,000 produced every year. Wetsuits, board shorts, and board wax are all composed of harmful materials including neoprene, nylon, and paraffin; and a wide range of products that are produced for the surf industry may be potentially damaging to the environment (Davis, 2009). Additionally, surfers may travel long distances to reach the majority of popular surfing areas, requiring long journeys in motor vehicles (Davis, 2009). There is therefore a conflict of interest between these essential but environmentally damaging factors of the surfers' lifestyle and their need to engage in proenvironmental behavior to protect their lifestyle.

Steg and Vlek (2009) argued that the quality of public transport systems, the availability and pricing of goods on the market, and access to local recycling facilities were all factors that influence proenvironmental behavior. Schultz and Zelezny (1999) have suggested then that the intent to engage in proenvironmental behavior is affected by an awareness of consequences. Additionally, Hansla, Gamble, Juliusson, and Gärling (2008) state that people are most likely to take part in proenvironmental behavior when they believe 


\section{HOLLAND-SMITH ET AL.}

the environmental problems have threatening and harmful consequences for objects they value.

When trying to predict proenvironmental behavior, attitudes have been demonstrated to be more influential than objective knowledge of environmental issues (Axelrod \& Lehman, 1993). However, the relationship between proenvironmental behavior and attitude is extremely complex, and researchers have moved away from focusing on the individual's attitudes to looking at the social construction of attitudes and how individuals' attitudes are a product of the social environment and context.

Cialdini, Kallgren, and Reno (1991) have hypothesized in the Theory of Normative Conduct that behaviors are determined by whether a person is focused on the norms of a culture, the situation (social contextual norms), or the self (personal norms). Sports such as surfing have been defined by Tomlinson, Ravenscroft, Wheaton, and Gilchrist (2005) as lifestyle sports. According to Tomlinson et al. (2005), lifestyles are patterns of actions that both define and differentiate people in terms of such aspects as their association with subcultures, patterns of behavior, and affiliations that they make. Affiliations become primary sources for social identity formation and can define, as in this case, what it is and means to be a surfer. Rinehart (2000) suggests that the practices and norms associated with particular subcultural groups are best understood by and through the meanings, values, and identities that are constructed from within by the people who belong to them and participate in them.

According to Heywood and Montgomery (2008), surfing is not just about the activity of surfing. It is also about the location, environment, and space in which surfing takes place, and surfers are unable to differentiate or separate out the act of surfing from the environment. Clayton (2003) argues that people who have strong environmental identities are more likely to demonstrate a proenvironmental attitude. Therefore surfers who experience total immersion in activities may be "galvanized" and more likely to participate in environmental activism as a result. This study therefore aims to expand the understanding of how surfers' identities and lifestyle influence their attitudes and environmental behaviors, focusing on the specific values, meanings, and attitudes of the people who identify themselves as being surfers from an insider's perspective.

\section{Methodology}

\section{Participants and recruitment}

The study was approved by the University's Research and Ethics AU1 Committee before potential participants were contacted. Eight participants were selected at random from an initial cohort of 50 surfers drawn from a variety of surfing locations and backgrounds. This included both male and female members, aged between 18 and 40 years, of British university surf clubs, surf instruction companies, surf shops and commercial outlets, as well as recreational surfers from various locations across the UK. All 50 participants were asked to complete a general questionnaire exploring their relationship and attitudes toward the environment. The questionnaire contained a mix of both closed and open questions exploring the background and surfing experience of the participants and specific attitudes and issues related to the environment. The purpose of this questionnaire was to establish some of the key issues and themes to be explored in depth through semi-structured interviews.

\section{Procedures}

Participants were informed of the nature and purpose of the research, how data would be used, and that it would be anonymized. They were also informed that they could end the interview or withdraw from the research at any point. All participants then signed an informed consent form. Interviews were conducted in a range of private locations, and each interview lasted between 30 and 60 minutes. Participants were encouraged to talk about and discuss their involvement in surfing, with the interviewer acting as an "active listener," (Sparkes, 2000) discussing and helping the participants explore their attitudes and beliefs without following a preconceived and rigid set of knowledge structures.

\section{Interview schedule}

The open questions from the initial questionnaire were analyzed and categorized as they were collected. Repetitions in the answers provided categories, which were then developed into themes. These themes related to general surfing background experience and involvement; their attitudes and relationship with the environment; proenvironmental behavior; specific issues, concerns, and behaviors related to surfing including transport, pollution, equipment, and commercial influences. The themes provided a general template to guide the interview. This followed a grounded approach as advocated by Glaser and Strauss (1967), which in turn allowed themes and issues to emerge from the research process and to generate theory.

\section{Data analysis}

The second author read the transcribed interview data and made notes in order to develop an understanding of the issues from the perspective of the participants (Maykut \& Morehouse, 1994). The data was then analyzed using the constant comparison method as outlined by Glaser and Strauss (1967). From this, emerging categories and themes were identified and organized into broader and more 


\section{SURFERS’ ATTITUDES TOWARD THEIR ENVIRONMENT}

general themes. The initial level of analysis involved using the direct quotations from the transcribed interviews to create themes. The relevant quotations taken from the participants were used as illustrative examples. Specific segments of text were extracted, coded, and gathered together under the identified themes. Once the initial themes had been identified, connections were made between themes and particular theoretical perspectives.

The second researcher was a regular surfer, and he closely identified with the surfing culture. This close association enabled him to probe and explore the issues in more depth. However, there was also an awareness that this could impact on the interpretation and analysis. Within qualitative research, the validity of the data is problematic, and there is a requirement to demonstrate the trustworthiness of the data and its analysis (Sparkes, 2000). There was the potential for the second researcher to become too close to the participants and make assumptions regarding the emerging issues and analysis (Hammersley \&t Atkinson, 2007). The first and third researchers acted as critical discussants and examined the emerging analysis while encouraging reflection and critical engagement. Their role was to ask probing theoretical questions of the data, the emerging themes, and interpretations (Hammersley \& Atkinson, 2007). Techniques such as interpreter reliability can provide high levels of credibility, but this can also be problematic, as it may lead to a trivialization of the interpretations being made (Kavale, 1996). Themes were therefore also reviewed by participants in order to ensure that the themes were an authentic representation. Following the analysis, participants were also invited to comment on draft versions of the analysis and results.

\section{Results}

Conditional proenvironmental behavior

The majority of participants expressed and discussed concerns for similar environmental issues. While many of them felt that their behavior was affected by their concern about the environment, it was also indicated, by their responses, that there were conditional or alternative reasons for their proenvironmental behavior. Generally participants were supportive of proenvironmental action and behavior as long as it did not impact upon the surfing culture and experience. An example of this was the conditional proenvironmental issues that were related to car use and renewable energy. The majority of the participants agreed that car sharing had a positive impact on the environment, but they also discussed reasons such as cost for their behavior, which shows that they were not motivated solely to be environmentally friendly purely or out of concern for the environment:
I have a few guys I surf with so we car share as much as possible. We live a good 45 minutes from the beach so it's cheaper to share the fuel and means we all aren't taking the car.

The participants also agreed that renewable energy was a positive idea and believed that it should be developed further. However, they were wary of the possible effects on both the environment and their own sport participation:

Renewable energy is a good idea but I don't want it to affect where I surf. There are also problems with its effect on the ecosystem and any project would need to address that issue.

I am really worried about the big push towards the tidal power and renewable energy. I think a lot of people don't realise that although these plans are great in theory, they will also cause a lot of adverse affects. The local ecosystem would suffer as a result of some of the methods and you can't replace one problem with another one, it makes no sense.

These statements from the participants show that they are interested in seeing changes in the way that energy is manufactured as long as this change does not affect their experience or threaten the existence of their sport and the environment in which it takes place.

\section{Mutual surfing and environmental benefits}

(pollution, environmental quality, and health)

Water quality was viewed as an important issue for all participants. Their responses on the issue of water quality revealed a bias in that they all discussed the issue from a water user's point of view such as how water quality could potentially cause them health problems and negatively affect their experience. However, only one participant discussed how this issue could have a major effect on the local ecosystem and how water that was not safe for humans to swim in also had a major effect on the wildlife.

The nuclear power plants have their water cooling outflow systems, that's not good and the water companies are bad for dispensing their wastewater. And that's just the effect on humans, you really get concerned about the effect that these things must have on the wildlife.

Water quality was highlighted as a major issue, as participants felt they were on the front line, as it were, and as such are acutely affected by the water quality. Many described feelings of their health being on the line, with statements such as "the sea is sick, I get sick" and "you get a sore throat sometimes after sessions." Issues such as these may 


\section{HOLLAND-SMITH ET AL.}

make water quality issues the biggest environmental concern for surfers:

Well my friend almost had his balls removed after surfing. It was polluted with farming fertilisers and pesticides. Don't think he enjoyed that! It's just horrible thinking about what you're surfing in.

The participants also discussed how many surfers want to see a change in the quality of the water, as it directly affects them as water users. Every participant reported negative experiences that had resulted from the quality of the water and also a feeling that they could not bring about a change on their own but needed others to realize the negative impact many of their behaviors were having:

Plastics are a major bug bear too. People rely on it and use it far too much. Then they just discard it without thinking about where it will end up. You see the effects of it as a water user, you sometimes see it floating past you, it washes up on the shore. I think people would change their ways if they spent time in the sea or even walked down at the beach more often!

\section{Personal environmental action and recycling}

The participants all stated that they were actively engaged in more AU1 general proenvironmental behaviors such as recycling (glass, paper/ cardboard and plastics), car sharing, and using low-impact materials:

I recycle everything that I can, most of it is picked up by my council, but the plastics have to be taken to the supermarket. I cycle as much as possible, and I car share whenever I go surfing. I'm lucky that I live near a few of my friends that surf, so we take turns to drive down. I don't use plastic bags anymore and I do bits and pieces throughout the house too such [energy efficient] light bulbs, washing at 30 degrees etc.

All the participants also recognized the problem with traditional surfboards and either already owned an eco-friendly board or said they would most likely buy one the next time they had to replace their current surfboard. The participants also stated that they were more likely to buy an eco-friendly surfboard because they had now been shown to perform just as well as other surfboards and the cost of ecofriendly surfboards had decreased substantially:

I also am in the market for a new board and I've been pricing and looking at the eco-friendly ones. There are lots of horrible things in the older boards and I definitely will make the switch now that I need a new board.

The respondents all participated in some form of environmentally responsible behavior; however, it is noticeable that much of their behavior was inconsistent and consisted of both positive and negative approaches to the environment that potentially cancel each other out.

\section{Surfers' relationship with the environment}

All the participants discussed a belief of being at one with the sea when they surfed. They described feelings and emotions that related to the ideas of total immersion. Many of them also discussed a feeling of water users being like guardians of the sea. They argued that it was their responsibility to look after the beach and sea and provide a voice for its welfare.

I think that surfing gives you a respect for the environment that you can't get from a lot of other activities. You feel like you are part of the ecosystem, and you really appreciate the true power of the sea... because of feeling like that, I think surfers feel they have a responsibility to respect and look after the ecosystem. But just changing your own lifestyle isn't enough...you have to add your voice to the campaigns and put some pressure on people to change, because if they don't change your efforts are in vain.

All the participants agreed that they could not give up their sport and saw no environmental benefit to considering such an idea: "No I wouldn't quit surfing. I don't see how my quitting would make a positive difference to the environment." They all described a feeling that they felt they could not experience in any other activity that they have experienced.

No I wouldn't [give up surfing]. It's such a powerful sport, the emotions that you feel when you're in the water. I think any surfer would tell you the same. You lose yourself when you are out on the water, nothing else matters and you get a real feeling of connection with the elements.

This response is another example of the feeling that many surfers describe. They feel that they are part of nature when they surf, and they often describe feeling "at one" with the sea. This could potentially be exploited by environmental groups; it is possible that more surfers could relate to the message and volunteer to protect the beach and sea environment:

Maybe if I didn't drive to the beach I'd not cause as much pollution but then would I be as bothered by the state of the sea? I think that I can make some changes and I am willing to do my bit, but I think that it's more important for me to keep surfing and keep an interest in the welfare of the sea and beach. 


\section{SURFERS’ ATTITUDES TOWARD THEIR ENVIRONMENT}

\section{Surfing-specific environmental action}

Although not all the participants were members of the British surfing environmental group Surfers Against Sewage many had donated or taken part in some of their campaigns:

I have also sent donations to [Surfers Against Sewage] and taken part in a couple of the campaigns. I haven't joined the group, but I do try and keep an eye on what they are doing and supporting them when the campaigns are at my beach.

However, those participants who were members of the organization expressed a far more proactive opinion about engaging in proenvironmental behaviors and the need to present a unified front in regard to the environmental concerns of surfers:

I am a member of [Surfers Against Sewage] and I take part in a lot of the campaigns that they organise. I think more people need to get involved though...people [need] to join up and add their voice.

The participants felt that taking part in local campaigns and giving their backing when it related to them was sufficient and helped them make a difference, while those that had signed up to the group felt that they needed the support and backing of more water users and desired more people to join and take part in more campaigns.

\section{Discussion}

The major proenvironmental behavior that all the participants claimed to participate in was recycling. Each surfer claimed that he or she recycled paper/cardboard, glass, and plastic on a weekly basis. While they described this behavior, they also played down the behavior as just something that everyone either does or should do. One

AU2 of the participants also mentioned that they felt it was becoming socially unacceptable not to recycle. This feeling among surfers points to the influence of social norms on their behavior and the theory of normative conduct (Cialdini et al., 1991).

Dunlap, Gallup, and Gallup (1993) suggest that the majority of the population in Western society is aware of the effect that humans have on the environment and that this population often displays a level of concern in regard to environmental problems. In spite of this fact, Seguin, Pelletier, and Hunsley (1998) state that it is extremely difficult for environmental groups to recruit volunteers and active members. This sentiment was also expressed by the participants in the present study. While many of the surfers discussed their participation in events organized by environmental pressure groups, only three of the eight respondents admitted to membership of an environmental pressure group. Public opinion has shifted in recent years, and people have a far more individualistic attitude toward the environment (Macnaghten, 2003). The public are not generally interested in political engagement and are more concerned about the environment's effect on their well-being and their lifestyle (Macnaghten, 2003).

The general consensus within the proenvironmental behavior literature is that there exists a wide spectrum of factors that lead to proenvironmental behavior. While concern for the environment may play a role in people's behavior, the greatest reasons for engaging in these behaviors often stem from other motivations and contextual factors such as cost saving or convenience (e.g., Bamberg \&t Schmidt, 2003). When asked to describe their proenvironmental behaviors and the reasons that led them to behave in that manner, the participants responded with a diverse range of reasons that they felt influenced their behavior, such as protection of their health, the quality of the AU1 surfing environment, water quality, and cost. While all the participants were keen to stress the fact that their concern for the environment made a major contribution to their proenvironmental behavior, they all went on to discuss further reasons for the positive behavior, including the impact of that behavior on their experience and social expectations.

According to Stern (2000), proenvironmental behavior is extremely difficult to predict and does not necessarily require environmental concern as a prerequisite. For example, Whitmarsh (2009) argued that the motivation for energy conservation was more often due to financial and health benefits than genuine concern for the environment. The participants in the current study discussed examples of times when they behaved in an environmentally responsible manner, and they also expressed their concern about environmental problems. However, these concerns were also followed by other motivations that influenced their behavior, such as the financial implications and the feeling of social expectations.

It was clear from the responses that the participants felt they were acting in a proenvironmental manner regularly. All of them stated that they recycled as much as they could and also car shared whenever possible. However, surfers are known to travel vast distances in search of the perfect surfing conditions. This is an illustration of one of the major contradictions that surfers face when they claim to be environmentally responsible. While they try to behave responsibly toward the environment, the reality for many surfers is that the long car journeys needed to maximize their surfing experiences may still cause considerable pollution.

Four of the eight participants discussed their willingness to change their boards from the toxic and non-biodegradable surfboards to the more environmentally friendly alternatives. While half the surfers 


\section{HOLLAND-SMITH ET AL.}

stated that they either had purchased or were willing to purchase an eco-friendly board, the reasons for their doing so were not purely motivated by environmental concern. Steg and Vlek (2009) have argued that when people make informed decisions about environmentally friendly products they choose between the products in regard to such factors as the greatest benefit versus lowest cost. While all participants mentioned the harmful ingredients of the old-style boards and their negative effect on the environment, the surfers all mentioned the cost of these eco-friendly boards being reasonable and the performance of the eco-friendly boards matching the performance of the alternatives.

When the participants were asked whether or not they would be willing to give up the sport in order to save the environment, their answers provided an insight into their regard for their sport. While the respondents were universal in their opinion that they would not be able to give up their sport, many of the surfers gave responses that indicated that they felt the sport was a part of their life. They indicated that they felt the act of surfing defined them and was a major part of their identity. Self-identity has been shown to give people an individual identity while shaping the way that they behave in response to the social group that they associate themselves with (Christensen et al., 2004). Charng, Pillavin, and Callero (1988) suggest that attitude may be overridden by identity in situations where behavior is dictated by role identity, even when the person may not like the behavior he or she expresses. This may also be the case with the participants in the current study. The participants stated that they have an identity that is environmentally friendly and therefore may, by defining themselves as surfers, feel obliged and willing to behave in the way which is dictated by their identity.

For the participants to give up surfing would call into question their identity. Being a surfer defines them as a person, and this identity shapes the way that they think and behave (Sparks \& Shepherd, 1992). This is potentially valuable to environmental pressure groups in their objective to gather support to their cause, as they can shape their campaigns so that surfers can relate to their mission. Potentially, if environmental groups were to focus on recruiting through group affinity and individual identity, then it may be the case that they can obtain a greater level of support than previously achievable. Macnaghten (2003) stated that the traditional methods of activism alienate the majority of the population, as people are more interested in the problems that will affect them than problems that are distant and irrelevant to them.

As well as defining themselves as surfers through their participation in the sport, the participants also discussed a feeling of being "at one" with the sea. This feeling is described as feeling a part of nature and the sea; rather than surfing on the wave, these participants felt like they were a part of the wave. Many of the participants also described their inability to separate the act of surfing from the location that they surfed at. They felt that without the place, the surfing couldn't exist; therefore, surfing was not only the sport but also the location. This indicates that the participants' identities were rooted in the act of surfing and the location at which they surf.

The participants mentioned that they felt they were responsible for the sea and the beach's ecosystem. This was apparent as many of the respondents discussed feeling like they had a duty to take steps toward protecting the areas that they surfed at. Place identity (Proshansky et al., 1983) can lead people into protective action in order to help protect and save certain places/ecosystems that they identify with. Clayton (2003) argued that a relationship exists between environmental identity and proenvironmental behavior such as recycling and energy efficiency, while Stets and Biga (2003) suggested that people who had a stronger environmental identity had a far more positive attitude toward the environment than those who had less environmental identity.

The benefit of understanding the attitudes of surfers toward the environment is vital for both the environment and environmental pressure groups. Through understanding what concerns this group of outdoor enthusiasts have, new campaigns can be developed to motivate them and promote proenvironmental behavior. It seems clear that surfers are environmentally aware and try to take steps toward improving their impact on the environment. However, while all of those interviewed recycled, had made changes to their purchasing behavior, and had tried to use their cars less, none of the surfers were able to contemplate giving up their sport even if it would directly improve the environment. This is one of the contradicting behaviors that not only typifies surfing but also is indicative of the general public's attempts to lessen their impact on the environment (Steg $\mathrm{Ct}$ Vlek, 2009). The participants felt that their identity as surfers placed an expectation on them to behave in an environmentally responsible manner. However, while the participants expressed their concern about environmental problems as being a major influence on their behavior, this was always conditional on such factors as social pressure, cost implications, the availability of services, the quality of products, and the surfing experience.

Future research should concentrate on exploring the question of when personal benefits outweigh the responsibilities of environmental stewardship. A future focus should also be on the intra- and interperspective of the surfing identity, as well as that of other outdoor enthusiasts, the links this has with the environment, and how environmental groups use this to gather support for their campaigns. 


\section{REFERENCES}

Axelrod, L., \& Lehman, D. (1993). Responding to environmental concern: What factors guide individual action? Journal of Environmental Psychology, 13, 149-159.

Bamberg, S., \& Schmidt, S. (2003). Incentives, morality, or habit? Predicting students' car use for university routes with the models of Ajzen, Schwarts, and Triandis. Environment and Behavior, 35, 264-285.

Charng, H., Pillavin, J., \&t Callero, P. (1988). Role identity and reasoned action in the prediction of repeated behavior. Social Psychology Quarterly, 51, 303-317.

Christensen, N., Rothberger, H., Wood, W., \&t Matz, D. (2004). Social norms and identity relevance: A motivational approach to normative behavior. Personality and Social Psychology Bulletin, 30, 1295-1309.

Cialdini, R., Kallgren, C., \& Reno, R. (1991). A focus theory of normative conduct: A theoretical refinement and reevaluation of the role of norms in human behavior. Advances in Experimental Social Psychology, 24, 557-572.

Clayton, S. (2003). Environmental identity: A conceptual and an operational definition. In S. Clayton \& S. Opotow (Eds.), Identity and the natural environment (pp. 45-66). Cambridge, MA: MIT Press.

Davis, H. (2009). Environmental issues in surfing-behaviours and attitudes. The Plymouth Student Scientist, 2, 108-155.

Dunlap, R., Gallup, G., \& Gallup. A. (1993). Of global concern: Results of the Health of the Planet survey. Environment, 35, 6-17.

Glaser, B., \& Strauss, A. (1967). The discovery of grounded theory: Strategies for qualitative research. Chicago, IL: Aldine.

Hammersley, M., \& Atkinson, P. (2007). Ethnography: Principles in practice. London: Routledge.

Hansla, A., Gamble, A., Juliusson, A., \&t Gärling, T. (2008). The relationships between awareness of consequences, environmental concern, and value orientations. Journal of Environmental Psychology, 28, 1-9.

Heath, H., \& Cowley, S. (2004). Developing grounded theory approach: A comparison of Glaser and Strauss. International Journal of Nursing Studies, 41, 141-150.

Heywood, L., \& Montgomery, M. (2008). Ambassadors of the last wilderness? Surfers, environmental ethics and activism in America. In M. Atkinson \& $\mathrm{K}$. Young (Eds.), Tribal play: Subcultural journeys through sport. Research in the sociology of sport (pp. 153-172). Bingley, UK: Emerald Group Publishing.

Kavale. S. (1996). Interviews. London, UK: Sage.

Macnaghten, P. (2003). Embodying the environment in everyday life practices. The Sociological Review, 51, 63-84

Maykut, P., \& Morehouse, R. (1994). Beginning qualitative research: A philosophic and practical guide. London: The Falmer Press.

Midol, N., \& Broyer, G. (1995). Towards an anthropological analysis of new sport cultures: The case of whiz sports in France. Sociology of Sport Journal, 12, 204-212.

AU4 Murray, P. (1999). Fundamental issues in questionnaire design. Accident \&t Emergency Nursing, 7, 148-153.
Proshansky, H., Fabian, A., \&t Kaminoff, R. (1983). Place-identity: physical world socialization of the self. Journal of Environmental Psychology, 3, 57-83.

Rinehart, R. E. (2000). Emerging arriving sport: Alternatives to formal sports. In J. Coakley \& E. Dunning (Eds.), The handbook of sports studies (pp. 504-519). London, UK: Routledge.

Schultz, P., \&t Zelezny, L. (1999). Values as predictors of environmental attitudes: Evidence for consistency across 14 countries. Journal of Environmental Psychology, 19, 255-265.

Seguin, C., Pelletier, L. G., \& Hunsley, F. (1998). Toward a model of environmental activism. Environment and Behavior, 30,628-652.

Sparkes, A. (2000). Illness, premature career-termination, and loss of self: A biographical study of an elite athlete. In R. L. Jones \& K. M. Armour (Eds.), Sociology of sport: Theory and practice (pp. 13-32). London, UK: Addison Wesley Longman.

Sparks, P., \& Shepherd, R. (1992). Self-identity and the theory of planned behavior: Assessing the role of identification with green consumerism. Social Psychology Quarterly, 55, 388-399.

Steg, L., \& Vlek, C. (2009). Encouraging pro-environmental behaviour: An integrative review and research agenda. Journal of Environmental Psychology, 29, 309317.

Stern, P. (2000). Toward a coherent theory of environmentally significant behavior. Journal of Social Issues, 56, 407-424.

Stets, J., \&t Biga, C. (2003). Bringing identity theory into environmental sociology. Social Theory, 21, 284-297.

Tomlinson, A., Ravenscroft, N., Wheaton, B., \&t Gilchrist, P. (2005). Lifestyle sports and national sport policy: An agenda for research. London, UK: Sport England.

Wade, A. (2007). Surf nation: In search of the first fast lefts and hollow rights of Britain and Ireland. London, UK: Simon and Schuster.

Whitmarsh, L. (2009). Behavioural responses to climate change: Asymmetry of intentions and impacts. Journal of Environmental Psychology, 29, 13-23.

Address correspondence to: David Holland-Smith

School of Social and Health Sciences University of Abertay Dundee

$U K, D D 11 H G$

E-mail: D.Holland-Smith@abertay.ac.uk

Received: March 11, 2013 Accepted: April 26, 2013

AU1 Sentence ok as written?

AU2 Please use either he or she, depending on the identity of the surfer who is being quoted.

AU3 Proshansky et al., 1983, as found in the references, correct?

AU4 The following are in the reference list but are not found in the text: Heath \&t Cowley, 2004; Murray, 1999. Please reconcile. 